\title{
Localización y magnitud del terremoto de 1785 en Colombia calculadas a partir de intensidades macrosísmicas
}

\author{
Augusto Antonio Gomez-Capera ${ }^{1, *}$, Elkin de Jesus Salcedo-Hurtado ${ }^{2,3}$, Dino Bindi ${ }^{4}$, Jose Enrique Choy ${ }^{5}$, \\ Julio Antonio Garcia-Pelaez ${ }^{6}$ \\ ${ }^{1}$ Istituto Nazionale di Geofisica e Vulcanologia, sezione di Milano, Milan, Italia \\ ${ }^{2}$ Observatorio Sismológico y Geofísico de la Universidad del Valle, Cali, Colombia \\ ${ }^{3}$ Departamento de Geografía, Universidad del Valle, Cali, Colombia \\ ${ }^{4}$ Deutches Geo Forschungs Zentrum GFZ, Center for Early Warning, Potsdam, Alemania \\ ${ }^{5}$ Laboratorio de Geofisica, Facultad de Ciencias, Universidad de los Andes, Mérida, Venezuela \\ ${ }^{6}$ GEM Foundation, Pavia, Italia
}

\begin{abstract}
Resumen
El terremoto ocurrido el 12 de julio de 1785 en Colombia ha sido uno de los eventos sísmicos con mayor número de estudios macrosísmicos realizados a partir de documentos históricos. En la mayoría de estos estudios se le asigna un valor de VIII como intensidad máxima. Con base en el estudio de Salcedo Hurtado y Castaño Castaño (2011), y mediante el método de Bakun y Wentworth (1997) y la relación de atenuación de la intensidad macrosísmica propuesta por Gómez Capera y Salcedo Hurtado (2002), se calculó la magnitud $\mathrm{m}_{\mathrm{b}}=6,9 \pm 0,2$ y la localización del epicentro macrosísmico (4,78 latitud; -73,90 longitud) a $28 \mathrm{~km}$ de Bogotá. La validación de la relación de atenuación se realizó con los parámetros instrumentales del terremoto del 24 de mayo de 2008, localizado en la misma zona mesosísmica del sismo histórico aquí analizado.
\end{abstract}

Palabras clave: estudio de terremoto histórico, intensidad macrosísmica, parámetros del terremoto.

Calculation of the location and magnitude of the 1785 earthquake in Colombia based on macroseismic intensity data

\begin{abstract}
The earthquake that hit Colombia on July 12, 1785, is one of the seismic events with the highest number of macroseismic studies based on historical sources. Most of these studies have assigned a maximum intensity of VIII to the event. Following the study of Salcedo Hurtado and Castaño Castaño (2011), and applying the Bakun and Wentworth (1997) method and the macroseismic intensity attenuation proposed by Gómez Capera and Salcedo Hurtado (2002), we calculated the magnitude $\mathrm{m}_{\mathrm{b}}=6.9 \pm 0.2$ and the macroseismic epicentre at $28 \mathrm{~km}$ from Bogotá. The validation of the attenuation model was performed with the instrumental parameters of the earthquake occurred on May 24, 2008, in the same region of the historical earthquake studied in the present article.
\end{abstract}

Key words: Historical earthquake study, macroseismic intensity, earthquake parameters.

El territorio colombiano ha sido afectado en diferentes épocas históricas por terremotos destructivos que han ocasionado pérdidas de vidas humanas y económicas. Teniendo en cuenta que la mayoría de estos terremotos pertenece a la etapa preinstrumental, la cual se extiende hasta los albores del siglo $\mathrm{XX}$, cuando no existían redes sismológicas, se debe recurrir a los datos históricos como elementos principales para el estudio de la sismicidad de Colombia. La sismología histórica se desarrolla a partir del análisis de fuentes históricas de las cuales se obtiene información de tipo descriptivo sobre los daños causados por los terremotos (Ambraseys, 1971). Dicha información es analizada e interpretada a través de una escala de referencia (por ejemplo, la escala macrosísmica europea EMS98, Grünthal, 1998), y a partir del análisis de los datos relativos a la distribución de daños, expresados en términos de intensidades macrosísmicas, es posible modelar la localización, la magnitud y las incertidumbres de dichos parámetros de la fuente del terremoto, que son decisivos en el cálculo de la amenaza y el riesgo sísmico en el territorio nacional.

\footnotetext{
*Correspondencia:

Antonio Gómez Capera, antonio.gomez@ingv.it

Recibido: 17 de febrero de 2014

Aceptado: 4 de junio de 2014
} 
Varias agencias nacionales e internacionales (Centro Regional de Sismología para América del Sur, Ceresis; Servicio Geológico Colombiano, SGC; GEM Foundation) tienen disponibles catálogos y bases de datos en línea con las intensidades macrosísmicas para sismos históricos y recientes, y en la literatura especializada también se encuentran estudios de diferentes autores (INGEOMINAS, 1999a; Espinosa Baquero, 2003; Cifuentes y Sarabia, 2011; Servicio Geológico Colombiano - Universidad Nacional de Colombia, SGC-UN, 2012). En las referencias citadas se constata que desde las épocas de la conquista y la colonia española hasta el siglo XXI, el nivel de sismicidad de
Bogotá es alto y la ocurrencia de sismos es irregular (Figura 1). A pesar de que el último sismo que ocasionó daños parciales en algunos sectores de la ciudad ocurrió en 1917, el Ceresis (1985) destaca información sobre terremotos fuertes ocurridos entre los años 1500 y 1981 en Colombia (Giesecke, et al., 2004); en la Figura 2 se muestran aquellos que ocasionaron efectos y daños en la ciudad de Bogotá. Específicamente en el periodo preinstrumental, se observa que de acuerdo con la interpretación hecha por Timoleón Palencia y Rafael Goberna (código TPRG en Figura 2; Tabla 1), cuatro grandes terremotos históricos $(1743,1785,1826$, 1917) impactaron la ciudad de Bogotá con intensidades de 8

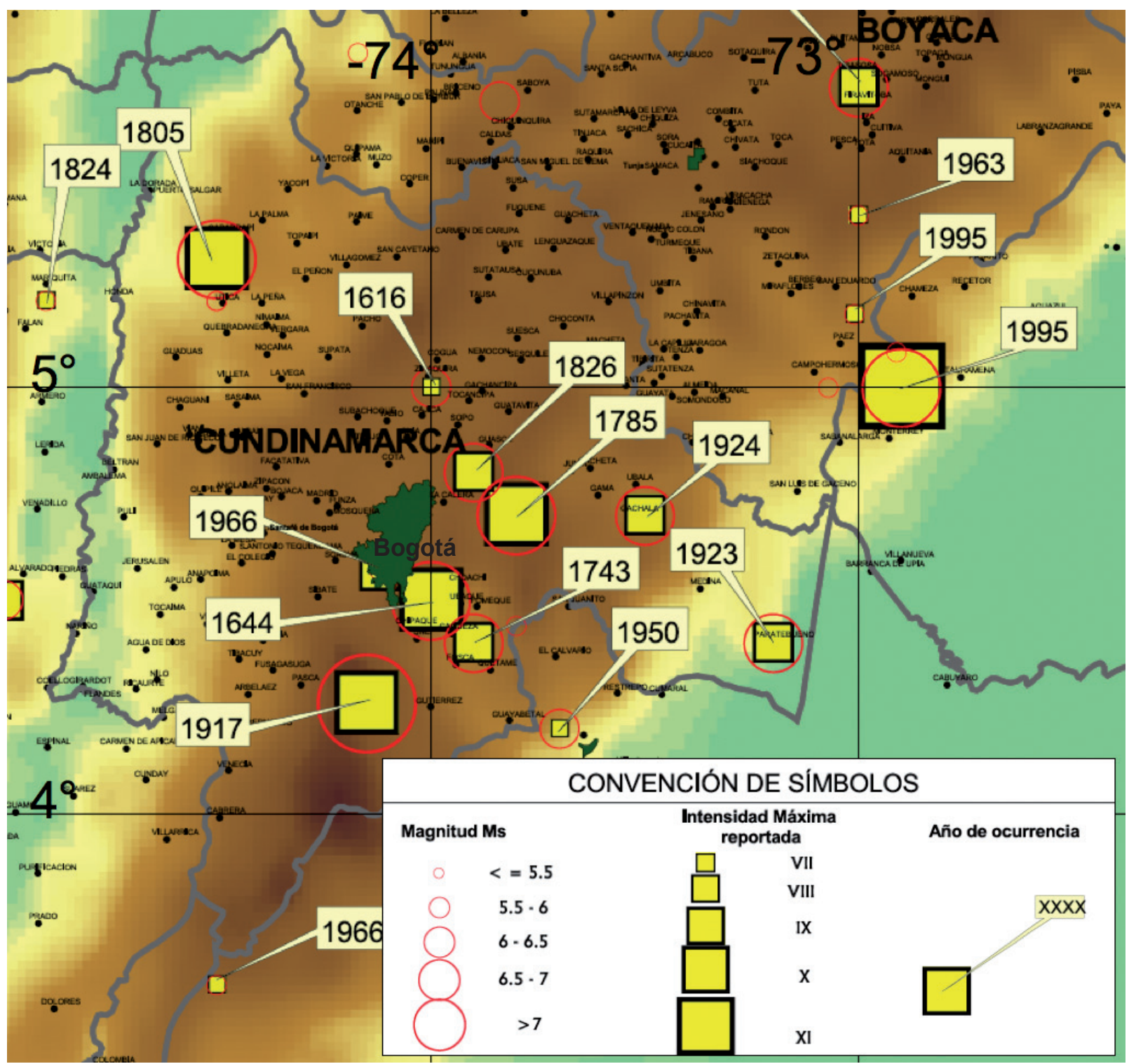

Figura 1. Historia sísmica de la región central del territorio colombiano, incluido el terremoto del 12 de julio de 1785 (modificada de Ingeominas, 1999a). 

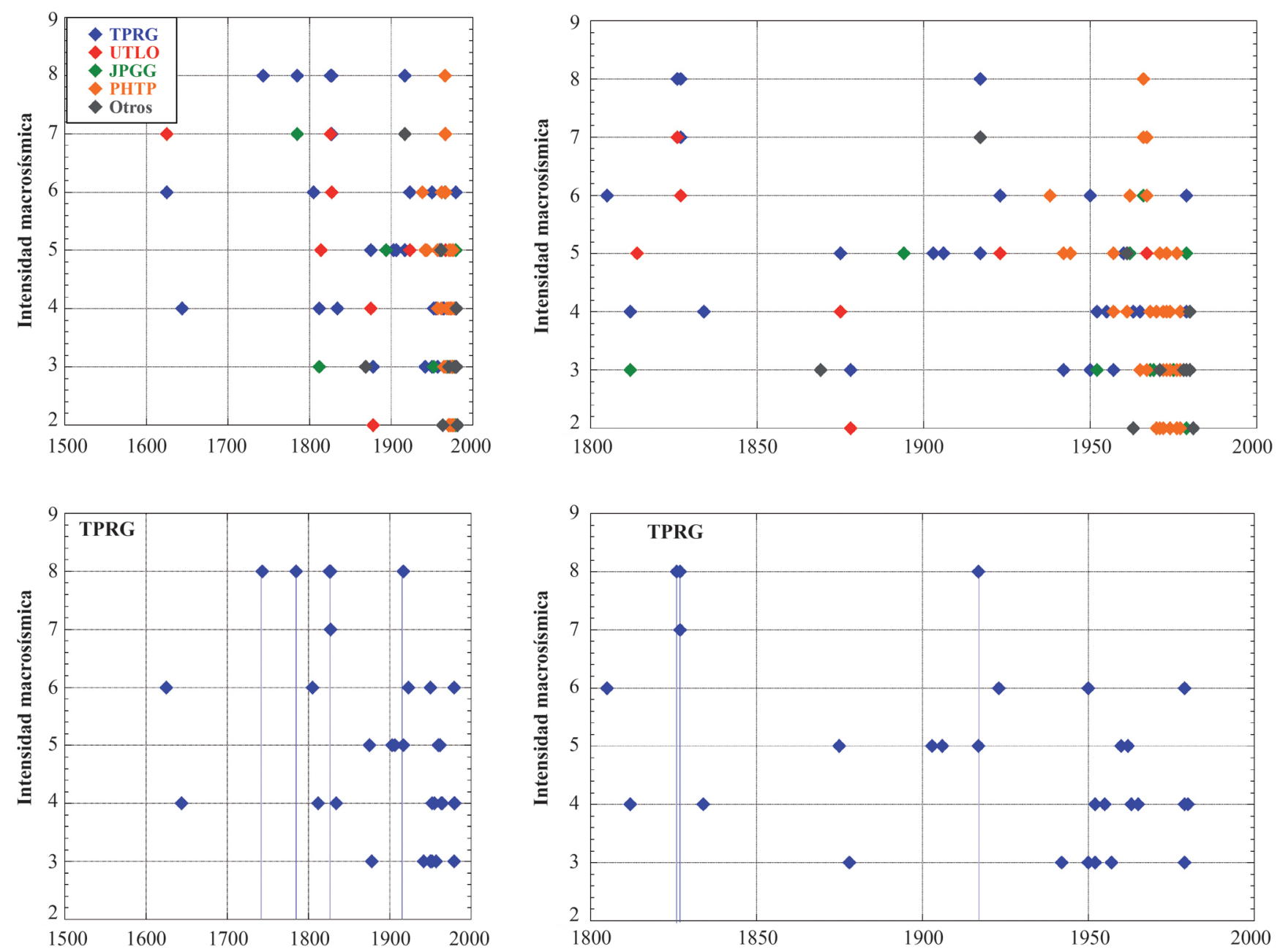

Figura 2. Historia sísmica de Bogotá, según el Ceresis (1985). Interpretaciones de intensidades dadas por TPRG: Timoleón Palencia y Rafael Goberna, del Instituto Geofísico de los Andes Colombianos, Universidad Javeriana, Bogotá; UTLO: Úrsula Torres y Leonidas Ocola, del Instituto Geofísico del Perú; JPGG: José Pablo Grases Galofré, de la Universidad Central de Venezuela; PHTP: Porfirio Huaco, del Instituto Geofísico del Perú, y Timoleón Palencia, y otros.

Tabla 1. Parámetros epicentrales para terremotos grandes históricos que han impactado la ciudad de Bogotá (según el Ceresis, 1985). No: número; Lat.: Latitud; Long.: Longitud; Ms: Magnitud de ondas de superficie; Io: Intensidad epicentral; MM: Escala de Mercalli Modificada.

\begin{tabular}{cccccccccccc}
\hline No. & Año & Mes & Día & Hora & Minutos & Segundos & Lat. & Lon. & Ms & $\begin{array}{c}\text { Io (MM) } \\
\text { Intensidad en } \\
\text { Bogotá }\end{array}$ \\
\hline 1 & 1743 & 10 & 18 & 15 & 45 & 0 & 4,50 & $-73,80$ & 6,5 & 9 & 8 \\
$\mathbf{2}$ & $\mathbf{1 7 8 5}$ & $\mathbf{7}$ & $\mathbf{1 2}$ & $\mathbf{1 2}$ & $\mathbf{4 5}$ & $\mathbf{0}$ & $\mathbf{4 , 7 0}$ & $\mathbf{- 7 3 , 8 0}$ & $\mathbf{6 , 5}$ & $\mathbf{8}$ & $\mathbf{8}$ \\
3 & 1826 & 6 & 18 & 3 & 45 & 0 & 4,80 & $-73,90$ & 7,0 & 8 & 8 \\
4 & 1827 & 11 & 16 & 22 & 45 & 0 & 1,80 & $-76,40$ & 7,0 & 10 & $7-8$ \\
5 & 1917 & 8 & 31 & 11 & 36 & 18 & 4,00 & $-74,00$ & 7,3 & 8 & 8 \\
\hline
\end{tabular}

MM (escala de Mercalli Modificada) y uno con intensidad de 7 a 8 MM en 1827 (Figura 2; Tabla 1). Salcedo-Hurtado y Gómez-Capera (2013) han propuesto la modelación de parámetros del terremoto de 1743 a partir de datos macrosísmicos. Siguiendo esta línea de estudio, el presente artículo se propuso hacer la modelación de la localización y la magnitud del terremoto del 12 de julio de 1785 usando los puntos de intensidad macrosísmica de dicho sismo propuestos por Salcedo Hurtado y Castaño Castaño (2011), así como el algoritmo de Bakun y Wentworth (1997) y la relación 
empírica de atenuación de la intensidad macrosísmica para terremotos con profundidades focales menores de $60 \mathrm{~km}$ en territorio colombiano propuesta por Gómez Capera y Salcedo Hurtado (2002).

\section{Estado del arte de los parámetros del terremoto del 12 de julio de 1785}

El estudio de Ramírez (1975) reporta que a las 7:45 a.m. (hora local) del 12 de julio de 1785 se produjo “el mayor y más destructor terremoto que experimentó Santa Fe y, en general, el virreinato en el siglo XVIII". Ramírez (1975) localiza este sismo en las coordenadas 4,70 $\mathrm{N}$ y 73,80 O, en las inmediaciones del municipio de La Calera, cerca de Bogotá; en dicho estudio no se asigna una magnitud al evento, pero sí un valor de intensidad en la triple escala III (equivalente a los grados IX, X, XI y XII de la escala MM). El catálogo del Ceresis (1985) reporta las mismas coordenadas epicentrales dadas por Ramírez (1975) y le asigna una magnitud MS de 6,5, la cual fue valorada e interpretada por Ramírez y su grupo de colaboradores del Instituto Geofísico de los Andes (Tabla 2). Hasta aquí, los parámetros evaluados para este terremoto responden a un "juicio de experto", pero las coordenadas epicentrales dadas por Ramírez (1975) se recogen en la literatura (Dimaté, et al., 1999; INGEOMINAS, 1999b y Espinosa Baquero, 2003). La magnitud Ms de 7,0 propuesta por INGEOMINAS (1999b) se calculó mediante la relación empírica (Gutenberg y Richter, 1956), es decir, Ms = (2/3) Io +1 , donde Io $=9 \mathrm{MM}$.

Espinosa Baquero (2003) también reporta este evento con una magnitud de 7,0 Ms. Gómez Capera y Leschiutta (2004) propusieron el primer estudio con el algoritmo de Gasperini, et al. (1999), y en la base de datos macrosísmica del Ceresis (1985) se recogen los parámetros de 37 terremotos superficiales determinados por este Centro para obtener una idea preliminar de las posibles fuentes de terremotos históricos en Sur América. Para el sismo de 1785, Gómez Capera y Leschiutta (2004) proponen una magnitud de momento (MW) de 6,3 a partir de los 17 puntos de intensidad fijados por el Ceresis (1985). A pesar de que el estudio citado sigue una metodología objetiva, el resultado fue muy preliminar, ya que el algoritmo de Gasperini, $\boldsymbol{e t}$ al. (1999), empleado en dicho estudio, fue calibrado para sismos superficiales de los Apeninos.

Dimaté, et al. (2005), reasignaron parámetros a este evento (6 $3 / 4-71 / 4 \mathrm{Mw}$ ) a partir de las relaciones obtenidas en su estudio entre la magnitud de momento $(\mathrm{Mw})$, la longitud de isosista de grado VIII y la longitud de ruptura (Dorbath, et al., 1990).

El Servicio Geológico Colombiano (SGC-UN, 2012) está trabajando en la asignación de parámetros a los terremotos históricos, y para el sismo de 1785 propone un epicentro (ad-hoc) y una magnitud de tipo intensidad macrosísmica de 6,8. La Tabla 2 muestra un resumen de los parámetros del terremoto de 1785 disponibles en la literatura.

\section{Datos macrosísmicos}

La intensidad macrosísmica responde a una clasificación empírica de la fuerza del movimiento del suelo medida con base en los efectos observados sobre la estructuras civiles, sobre el ambiente y sobre el hombre en un área limitada (Grünthal, 1998). La intensidad macrosísmica es intrínsecamente cualitativa y su dependencia con respecto a los efectos de fuente, a la propagación de las ondas sísmicas y a los efectos locales de sitio no son claras; sin embargo, y a pesar de los avances en la sismología instrumental, continúa jugando un papel importante en la comprensión de la actividad sísmica de una región.

El terremoto de 1785 en Colombia es uno de los eventos sísmicos más estudiados por diversos autores e instituciones con base en la investigación histórica, lo que ha resultado en una variedad de interpretaciones en torno a su intensidad macrosísmica (Tabla 3). La base de datos del Ceresis (1985) sobre Colombia, en especial sobre el terremoto de 1785, propone las interpretaciones de la intensidad dadas por tres grupos de autores (Tabla 3):

Timoleón Palencia y Rafael Goberna (TPRG), del Instituto Geofísico de los Andes Colombianos, Universidad Javeriana, Bogotá; Úrsula Torres y Leonidas Ocola (UTLO), del Instituto Geofísico del Perú, y José Pablo Grases Galofre (JPGG), de la Universidad Central de Venezuela.

Tabla 2. Parámetros del terremoto del 12 de julio de1785 dados por diferentes catálogos. Coordenadas del epicentro (Lat., Lon.); h: profundidad; Io: intensidad epicentral o máxima; MM: escala de Mercalli Modificada; MSK: escala MSK, *** Triple Escala. Mm: magnitud macrosísmica.

\begin{tabular}{|c|c|c|c|c|c|c|}
\hline Catálogo o estudio & Lat. & Lon. & $\mathbf{h}(\mathbf{k m})$ & Magnitud & Io & Escala \\
\hline Ramírez (1975) & 4,70 & $-73,80$ & - & - & III & $* * *$ \\
\hline Ceresis (1985) & 4,70 & $-73,80$ & - & $6,5 \mathrm{Ms}$ & 8 & MM \\
\hline Ingeominas (1999b) & 4,70 & $-73,80$ & - & 7,0Ms & 9 & MSK \\
\hline Espinosa Baquero (2003) & 4,70 & $-73,80$ & 30 & $7,0 \mathrm{Ms}$ & 9 & MSK \\
\hline Dimaté, et al. (2005) & 5,00 & $-73,71$ & - & $63 / 4-71 / 4 \mathrm{Mw}$ & 9 & MSK \\
\hline SGC-UN (2012) & 4,00 & $-74,00$ & 20 & $6,8 \mathrm{Mm}$ & 7 & EMS98 \\
\hline
\end{tabular}


Tabla 3. Estudios hallados en la literatura científica con puntos de intensidad macrosísmica calculados para el terremoto del 12 de julio de 1785 . N: número de puntos de intensidad macrosísmica

\begin{tabular}{lccc}
\hline \multicolumn{1}{c}{ Estudio macrosísmico } & N & $\begin{array}{c}\text { Intensidad } \\
\text { máxima }\end{array}$ & Escala \\
\hline Ceresis (1985) interpretado por TPRG & 16 & 8 & MM \\
\hline Ceresis (1985) interpretado por UTLO & 6 & 7 & MM \\
Ceresis (1985) interpretado por JPGG & 4 & 7 & MM \\
\hline Álvarez (1987) & 27 & 8 & MM y MCS \\
Espinosa Baquero (2003) & 27 & 8 & MSK \\
\hline Sarabia Gómez, et al. (2006) & 18 & 7 & EMS98 \\
Sarabia Gómez, et al. (2010) & 17 & 8 & EMS98 \\
Salcedo Hurtado y Castaño Castaño (2011) & 28 & 8 & EMS98 \\
SGC-UN (2012) & 18 & 7 & EMS98 \\
\hline
\end{tabular}

Los tres grupos asignan intensidades en la escala MM. El grupo TPRG le da 16 puntos de intensidad con valor máximo de $\mathrm{I}=8$. Álvarez (1987) propone 27 puntos de intensidades entre 5 y 8 en la escala MM y en la de Mercalli-CancaniSierberg (MCS), sin especificar qué valores se evaluaron en la MM y cuáles en la MCS. Espinosa Baquero (2003) propone 27 puntos de intensidad en la escala de MedvedevSponheuer-Karnik (MSK), también con un valor de 8 como intensidad máxima.

El Servicio Geológico Colombiano y la Universidad Nacional de Colombia realizaron una nueva investigación histórica en archivos nacionales y en diversos estudios han propuesto crear la base de datos de intensidad macrosísmica para el territorio colombiano (Cifuentes y Sarabia, 2011; SGC-UN, 2012). Con respecto al sismo de 1785 , se observan diferentes interpretaciones de las intensidades en 18 localidades medidas en dos escalas, la Escala Macrosísmica Europea (EMS98) y la escala de Mercalli Modificada (MM) (Sarabia Gómez, et al., 2006; Sarabia Gómez, et al., 2010; SGC-UN, 2012).

Salcedo-Hurtado y Castaño-Castaño (2011) proponen una reevaluación de las intensidades macrosísmicas asignadas a este evento sísmico en la escala EMS98 y ofrecen nuevas hipótesis de 28 puntos de intensidades macrosísmicas distribuidas a lo largo de la cordillera andina. La intensidad máxima ( $\mathrm{I}=8, \mathrm{EMS} 98)$ se le asignó a la localidad de Santa Fe (hoy Bogotá, D.C.) y a otras poblaciones como Cajicá, Chía, Engativá, Fontibón y Soacha, que actualmente son municipios del área metropolitana de la capital de la República; el sismo se sintió igualmente en Pasto y Popayán ( $\mathrm{I}=6$, EMS98) y el valor mínimo asignado es de $\mathrm{I}=5$ en las ciudades de Cali y Pamplona.

\section{El método de Bakun y Wentworth}

El método de Bakun y Wentworth (1997) (B\&W97), analiza los datos de la intensidad macrosísmica para determinar la localización y la magnitud de la fuente sísmica. El B\&W97 es apropiado para eventos históricos cuyos parámetros sísmicos son poco conocidos y por lo regular han sido asignados de manera subjetiva.

Desde el punto de vista físico, el método se basa en dos planteamientos centrales: a) para una profundidad focal dada, las intensidades decrecen con la distancia desde el epicentro, y b) para una distancia epicentral dada, la intensidad es función de la magnitud. El B\&W97 utiliza un modelo de fuente puntual, ubicada a una profundidad h, y un funcional empírico apropiado de atenuación de la intensidad macrosísmica, I, en función de la distancia y de la magnitud del terremoto, y adopta la siguiente forma:

$$
I_{i j}=f\left(M_{j}, R_{i j}\right)
$$

donde " $\mathrm{M}_{\mathrm{j}}$ " es la magnitud del $\mathrm{j}$-ésimo terremoto $\left(\mathrm{j}=1, \ldots \mathrm{N}_{\mathrm{eq}}\right.$ donde $\mathrm{N}_{\mathrm{eq}}^{\mathrm{j}}$ es el número de terremotos considerados), " $\mathrm{R}_{\mathrm{ij}}$," es la distancia hipocentral $\left[\left(\mathrm{R}=\left(\mathrm{x}^{2}+\mathrm{h}^{2}\right)^{1 / 2}\right]\right)$ para el j-ésimo terremoto sentido en el sitio "i", e " $\mathrm{I}_{\mathrm{ij}}$ " es la intensidad macrosísmica, siendo " $\mathrm{x}_{\mathrm{ij}}$ " la distancia epicentral del j-ésimo terremoto sentido en el sitio " $i$ " y "h" la profundidad de la fuente, que en la versión original los autores fijan en 10 $\mathrm{km}$. Para la región de interés, la función (1) se determina utilizando un conjunto de eventos cuyos datos macrosísmicos y parámetros de terremoto sean de alta calidad, es decir, sismos para los cuales se conozcan las magnitudes y las localizaciones instrumentales de manera precisa. Calibrando el método con estos datos y aplicándolo luego al evento sobre el cual no se tiene información instrumental, o esta no es confiable, se pueden obtener la localización y la magnitud equivalentes a dichas instrumentales.

Para el modelo de atenuación, el B\&W97 tradicionalmente usa una funcional que es lineal con la magnitud de momento y log-lineal con la distancia:

$$
I_{i j}=a+b M w-c R_{i j}-d\left(\log _{10} R_{i j}\right)
$$

donde "a", "b", "c", "d" son constantes que se calibran de acuerdo a la región donde se hace el estudio. En particular, "c" corresponde al coeficiente de atenuación anaelástico y "d", al coeficiente de atenuación geométrico (Howell y Schultz, 1975).

En el B\&W97 se aplica la ecuación (2) para modelar una magnitud MI calculada únicamente a partir de los datos de intensidad macrosísmica; dicha magnitud computada es equivalente a la $\mathrm{M}_{\mathrm{w}}$ si en el proceso de calibración se utilizan sismos con magnitudes en esta escala.

\section{Cómputo de los parámetros del terremoto}

En una primera fase, la ecuación (2) se invierte para calcular la magnitud individual en el sitio $\mathrm{MI}_{\mathrm{ij}}$, es decir, la magnitud asociada a cada punto de intensidad $\mathrm{I}_{\mathrm{ij}} \mathrm{j}$, con $\mathrm{i}=1, \ldots, \mathrm{P}_{\mathrm{j}}$, donde 
$\mathrm{P}_{\mathrm{j}}$ es el número total de puntos de intensidad disponible para el j-ésimo terremoto a partir de los valores individuales de intensidad $\mathrm{I}_{\mathrm{ij}}$ observados en distancias $\mathrm{R}_{\mathrm{ij}}$ :

$$
\mathrm{MI}_{\mathrm{ij}}=(1 / \mathrm{b})\left[\mathrm{I}_{\mathrm{ij}}-\mathrm{a}+\mathrm{cR} \mathrm{ij}_{\mathrm{ij}}+\mathrm{d}\left(\log _{10} \mathrm{R}_{\mathrm{ij}}\right)\right]
$$

En una segunda fase, mediante el B\&W97 se calcula la localización y la magnitud de un j-ésimo terremoto dado a partir del cómputo de $\mathrm{MI}^{\mathrm{k}}{ }_{\mathrm{ij}}$ sobre una malla de puntos de posibles epicentros localizados en $\mathrm{x}_{\mathrm{k}}\left(\right.$ latitud $_{\mathrm{k}}$, longitud $\left._{\mathrm{k}}\right)$. La magnitud $\mathrm{MI}^{\mathrm{k}}{ }_{\mathrm{j}}$ se define como el promedio de las magnitudes $\mathrm{MI}^{\mathrm{k}}{ }_{\mathrm{ij}}$ calculadas a partir de puntos de intensidad macrosísmica para el j-ésimo terremoto y asumiendo que el epicentro se localiza en la coordenada $\mathrm{x}_{\mathrm{k}}$,

$$
\mathrm{MI}_{\mathrm{j}}^{\mathrm{k}}=\frac{1}{\mathrm{P}_{\mathrm{j}}} \sum_{\mathrm{i}=1}^{\mathrm{P}_{\mathrm{j}}} \mathrm{MI}_{\mathrm{ij}}^{\mathrm{k}}
$$

Al considerar una malla de puntos de posibles epicentros $\mathrm{x}_{\mathrm{k}}$, las raíces medias cuadráticas $\left(\mathrm{rms}\left[\mathrm{MI}^{\mathrm{k}}\right]\right)$ se calculan como:

$$
\operatorname{rms}\left[\mathrm{MI}_{\mathrm{j}}^{\mathrm{k}}\right]=\left[\frac{1}{\sum_{i} \mathrm{w}_{i}^{2}} \sum \mathrm{w}_{i}\left(\mathrm{MI}_{i j}^{\mathrm{k}}-\mathrm{MI}_{j}^{\mathrm{k}}\right)^{2}\right]^{1 / 2}
$$

donde $\mathrm{w}_{\mathrm{i}}$ es una función peso con respecto a la distancia (Bankun y Wentworth,1997):

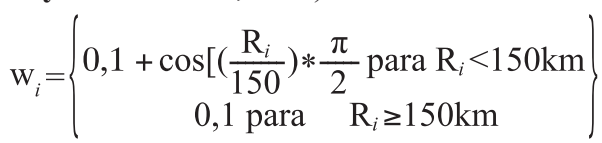

El mínimo de todas las rms en la malla se resta de cada una de ellas. La malla se toma con celdas de paso de 1,0 km. De acuerdo con Bakun y Wentworth (1997) y Bakun (1999), el centro de intensidades $(\mathrm{CI})$ corresponde a:

$$
\mathrm{rms}-\min (\mathrm{rms})=0
$$

donde la magnitud de intensidad $\mathrm{MI}_{\mathrm{j}}$ es dada por $\mathrm{MI}_{\mathrm{j}}^{\mathrm{k}}$ evaluada en el punto (latitud, longitud) que corresponde al CI.

El CI corresponde a la localización de la fuente puntual que mejor satisface los puntos de intensidad macrosísmica. A diferencia de la definición clásica de epicentro como el punto en la superficie de la Tierra que se encuentra sobre la proyección vertical del foco sísmico donde se inicia la ruptura, el método B\&W97 usa el CI como la localización del evento sísmico a partir de los puntos de intensidad macrosísmica, que en el presente estudio se adoptó como el epicentro macrosísmico. La magnitud MI en el CI es la magnitud equivalente a la $\mathrm{M}_{\mathrm{w}}$ del terremoto. Las incertidumbres en la determinación de la $\mathrm{M}_{\mathrm{w}}$ calculada están asociadas con el número de puntos de intensidad macrosísmica (NMDP) según Bakun y Wentworth (1999).

Los niveles de las rms corresponden a intervalos de confianza en la eventualidad de que el CI se encuentre dentro del área delimitado por ellas. Los niveles de rms usualmente se representan en el método de B\&W97 al 95, 90, 80, 67 y $50 \%$ a partir de valores tabulares publicados por Bakun y Wentworth (1999).

Esta metodología se ha aplicado en diferentes regiones como California (Bakun, 2006), Ecuador (Beauval, et al., 2010), Venezuela (Choy, et al., 2010), Suiza (Álvarez-Rubio, et al., 2011), Haiti (Bakun, et al., 2012), Asia central (Bindi, et al., 2013a, 2013b), sureste de Cuba (Gómez-Capera, et al., 2013) y en estudios de incertidumbres como el de Bakun, et al. (2011). En Colombia, el método de B\&W97 se ha aplicado al terremoto histórico de 1743, ocurrido en la región central andina (Salcedo-Hurtado y Gómez Capera, 2013), el cual impactó la colonial Santa Fe de Bogotá (Figura 1).

\section{Cálculo de los parámetros macrosísmicos}

El presente estudio empleó el modelo de atenuación de la intensidad macrosísmica publicado para Colombia por Gómez Capera y Salcedo Hurtado (2002) y expresada como:

$$
\mathrm{I}_{\mathrm{ij}}=\left(2,3 \mathrm{~m}_{\mathrm{b}}-6.8\right) 1.1 \mathrm{x}^{-0,06} \mathrm{e}^{-0,001 \mathrm{x}}
$$

donde $\mathrm{m}_{\mathrm{b}}$ es la magnitud de ondas de cuerpo, $\mathrm{x}$ es la distancia epicentral y los valores de los coeficientes de atenuación anaelástica y geométrica son de $(-0,001)$ y $(-0,06)$, respectivamente. El modelo de atenuación dado por la ecuación (8) está en función de la distancia epicentral, lo que hace que no dependa explícitamente de la profundidad focal. La relación empírica (8) se modeló con un conjunto de 24 terremotos con profundidades menores de $60 \mathrm{~km}$ y válida para $\mathrm{x}<400$ km (Gómez Capera, 1998; Gómez Capera y Salcedo Hurtado, 2002). El método B\&W97 aplica la ecuación (8) para calcular la magnitud $\mathrm{M}_{\mathrm{I}}$, que corresponde a una magnitud aproximadamente equivalente a la $\mathrm{m}_{\mathrm{b}}$. Se usó el CI como la localización de la fuente y se le adoptó como epicentro macrosísmico; la magnitud $\mathrm{M}_{\mathrm{I}}$ en el CI se adoptó como la magnitud del sismo.

\section{Prueba de validación}

En el presente estudio se realizó, además, un ejercicio de validación de la relación de atenuación dada por la ecuación (8) usando la siguiente información:

datos instrumentales del terremoto del 24 de mayo de 2008 (Tabla 4; Figura 3), localizado en cercanías de Quetame, en la misma zona mesosísmica del evento del 12 de julio de 1785, que determinaron su epicentro en 4,40 N y 73,81 O, su profundidad focal en $8,6 \mathrm{~km}$ (Red Sismológica Nacional de Colombia) y su magnitud en 5,5 m y 5,9 Mw (Global CMT), y puntos de intensidades macrosísmicas (Fig. 3), publicados por el Servicio Geológico y la Universidad Nacional de Colombia (SGC-UN, 2012; Sarabia y Cifuentes, 2009), evaluados en la escala EMS98 y en la MM. En el presente trabajo se tomaron las intensidades evaluadas en la EMS98. 
Tabla 4. Parámetros de catálogo Vs. parámetros macrosísmicos propuestos en el presente estudio. RSNC: Red Sismológica Nacional de Colombia. N: número de puntos de intensidad macrosísmica. Ix: intensidad macrosímica máxima en EMS98.

\begin{tabular}{|c|c|c|c|c|c|c|c|c|c|c|c|c|}
\hline \multicolumn{7}{|c|}{ Parámetros de catálogo } & \multicolumn{3}{|c|}{ Datos macrosísmicos } & \multicolumn{3}{|c|}{$\begin{array}{l}\text { Parámetros calculados } \\
\text { en el presente estudio }\end{array}$} \\
\hline Fecha & Lat. & Long. & $\begin{array}{c}\mathrm{H} \\
(\mathbf{k m})\end{array}$ & $\begin{array}{c}\text { Fuente } \\
\text { (hipocentro) }\end{array}$ & $\mathbf{M}$ & $\begin{array}{l}\text { Fuente } \\
\text { (M) }\end{array}$ & Estudio macrosísmico & $\mathbf{N}$ & $\mathbf{I x}$ & Lat_M & Lon_M & $\mathbf{m}_{b}$ \\
\hline 2008.05 .24 & 4,40 & $-73,81$ & 8,6 & RSNC & $\begin{array}{c}5,5 \mathrm{~m}_{\mathrm{b}} \\
5,9 \mathrm{Mw}\end{array}$ & CMT & $\begin{array}{l}\text { Cifuentes y Sarabia } \\
\text { (2009) }\end{array}$ & 21 & 8 & 4,34 & $-73,86$ & $5,6 \pm 0,2$ \\
\hline 1785.07 .12 & 4,70 & $-73,80$ & 30 & $\begin{array}{c}\text { Espinosa } \\
\text { Baquero (2003) }\end{array}$ & $7.0 \mathrm{Ms}$ & $\begin{array}{c}\text { Espinosa } \\
\text { Baquero (2003) }\end{array}$ & $\begin{array}{l}\text { Salcedo Hurtado y } \\
\text { Castaño Castaño (2011) }\end{array}$ & 28 & 8 & 4,78 & $-73,90$ & $6,9 \pm 0,2$ \\
\hline
\end{tabular}
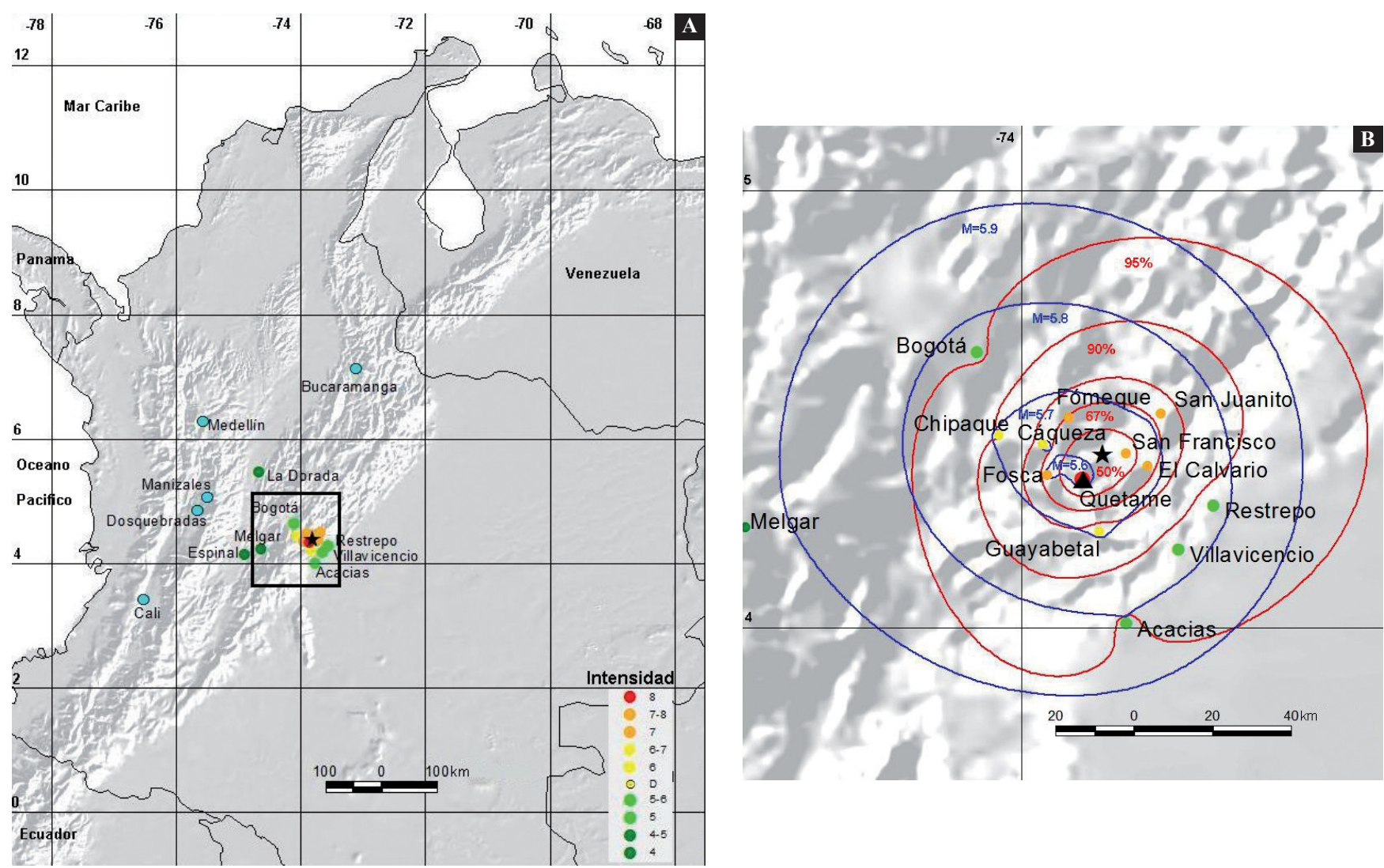

Figura 3. Terremoto del 24 de mayo de 2008 en Quetame. Puntos de intensidad macrosísmica tomados de INGEOMINAS (2009). Intensidad máxima en Quetame (I=8EMS98). Parámetros (Tabla 4): epicentro instrumental (estrella, 4,40N y 73,81O). Centro de intensidades adoptado como epicentro macrosísmico representado por el "triángulo" $(4,34 \mathrm{~N}$ y $73,86=)$ propuesto en el presente estudio. Magnitud instrumental: 5,5m (Global CMT). Magnitud calculada: $5,6 \mathrm{~m}_{\mathrm{b}}$. La isolíneas de color azul corresponden a los contornos de magnitud macrosísmica y las de color rojo a los contornos de $\operatorname{rms}\left[\mathrm{M}_{\mathrm{I}}\right]$ para los intervalos de confianza.

La intensidad máxima fue de 8 en la localidad de Quetame. En las localidades de El Calvario, Fosca, Fómeque, San Juanito y San Francisco la intensidad asignada fue de 7. La asignada a Cáqueza, Chipaque y Guayabal fue de 6, y en las ciudades de Villavicencio, Bogotá, Acacías y Restrepo, la intensidad asignada fue de 5 en la EMS98.
Los parámetros macrosísmicos calculados para este evento se resumen en la Tabla 4 y en la Figura 3b. No obstante, este fue un evento sísmico con un número discreto de puntos de intensidades $(\mathrm{N}=21)$ distribuidos de manera azimutal, y mediante la modelación macrosísmica se calculó el CI a 9 $\mathrm{km}$ al sureste del epicentro instrumental, con intervalos de 
confianza entre 50 y $95 \%$, y a $42 \mathrm{~km}$ al sureste de Bogotá. La magnitud calculada para el terremoto de Quetame del 2008 fue de 5,60, que corresponde a la isolínea azul que intercepta el epicentro macrosísmico, siendo ésta una magnitud equivalente $a \mathrm{~m}_{b}$ según la ecuación (8). La incertidumbre media es de $\pm 0,2$ en el $67 \%( \pm 1 \sigma)$ del nivel de confianza y 0,3 en el $95 \%( \pm 2 \sigma)$ del nivel de confianza (Bakun y Wentworth, 1999).

Para explorar la razón por la que se obtiene un valor razonable de la magnitud calculada a partir de los datos de intensidades, se pueden analizar los valores de la intensidad del sismo de Quetame del 2008 en función de la distancia. El decaimiento de las intensidades observadas (Sarabia y Cifuentes, 2009) con respecto a la distancia epicentral muestra, en general, un buen acuerdo con el modelo de atenuación de Gómez Capera y Salcedo Hurtado (2002) (Figura 4). La diferencia de 0,1 en la unidad de magnitud puede estar asociada al hecho de que la curva de atenuación tiende a anticipar los valores altos de intensidad para $\mathrm{x}<25$ $\mathrm{km}$ por efecto de la singularidad del modelo en distancias nulas, es decir, muy cercana de la fuente. Por otro lado, a distancias de $\mathrm{x}>100 \mathrm{~km}$, el modelo tiende a sobreestimar la intensidad en relación con las intensidades observadas. Sin embargo, se puede argumentar que la diferencia entre la magnitud obtenida en este trabajo y la instrumental fue inferior al intervalo de incertidumbre.

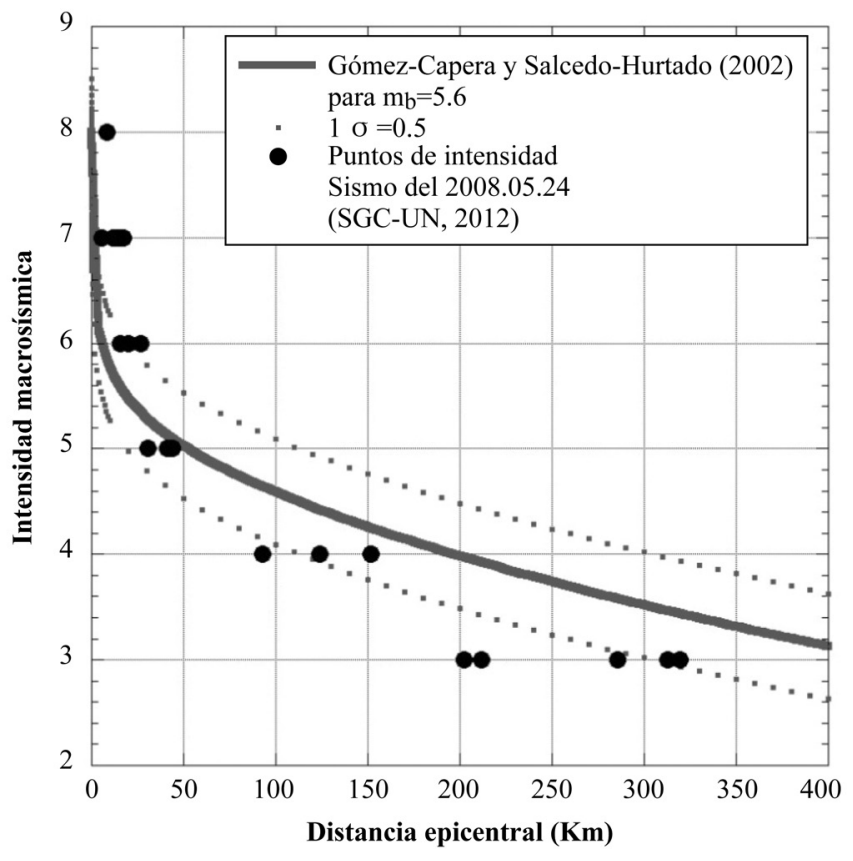

Figura 4. Intensidades macrosísmicas observadas en el terremoto de Quetane del 24 de mayo de 2008 con 5,5 mb , comparado con la curva de atenuación de Gómez Capera y Salcedo Hurtado (2002) para una 5,6 $\mathrm{m}_{\mathrm{b}}$ (línea sólida).
Parámetros del terremoto de $\mathbf{1 7 8 5}$ a partir de puntos de intensidades macrosísmicas

Los puntos de intensidad macrosísmica del terremoto del 12 de julio de 1785 usados para los cálculos y tomados de Salcedo Hurtado y Castaño Castaño (2011), se muestran en la Figura 5a. El resultado de la aplicación del método B\&W97 usando la ecuación (8), se presenta en la Figura 5b y en la Tabla 4. Las coordenadas del CI (4,66 N y 73,90 O), punto adoptado como epicentro macrosísmico, se encuentran a $28 \mathrm{~km}$ al noreste de Bogotá con una probabilidad del 0,50 y 0,80 de que el centro de energía se localice dentro de las líneas de contorno del 50 y el $80 \%$, respectivamente. Por otro lado, el CI se localizó a $14 \mathrm{~km}$ del epicentro propuesto por el Ceresis (1985) y Espinosa Baquero (2003) y siempre en la cordillera Oriental (triángulo, Figura 5b). La magnitud macrosísmica en el centro de intensidades obtenida (isolínea azul, Figura 5b) fue de $\mathrm{m}_{\mathrm{b}}=6,9$ con incertidumbre media de $2 \sigma(95 \%)$ de $\pm 0,30$ y $\pm 0,2$ para $1 \sigma(67 \%)$.

El decaimiento de las intensidades propuestas por Salcedo Hurtado y Castaño Castaño (2011) con respecto a la distancia epicentral (Figura 4) muestra un buen acuerdo con la curva de atenuación de Gómez Capera y Salcedo Hurtado (2002) (Figura 4). No obstante, en las distancias en las que $\mathrm{x}>250 \mathrm{~km}$ se observan sesgos que pueden asociarse a efectos de sitio y a las mismas dificultades implícitas en la asignación de intensidades en los casos de sismos históricos.

Comparación de $\mathrm{m}_{\mathrm{b}}$ y Ms de origen macrosísmico con Mw

Al comparar el resultado obtenido en el presente estudio para el sismo del 2008 en Quetame con la magnitud momento $(5,9 \mathrm{Mw}$, Global CMT; 5,87 $\pm 0,10$; Storchack, et al., 2013), Scordillis (2006) ha propuesto un modelo empírico aproximadamente lineal de Mw como función de $\mathrm{m}_{\mathrm{b}} \mathrm{y}$ Ms:

$\mathrm{Mw}=0,85( \pm 0,04) \mathrm{m}_{\mathrm{b}}+1,03( \pm 0,23), \quad 3,5 \leq \mathrm{m}_{\mathrm{b}} \leq 6,2$

$\mathrm{Mw}=0,99( \pm 0,02) \mathrm{Ms}+0,08( \pm 0,13), \quad 6,2 \leq \mathrm{Ms} \leq 8,2$

Cuando $m_{b}>6,2$, la relación (9) incrementa mucho la pendiente, mostrando un comportamiento inestable que podría considerarse como saturación (Scordillis, 2006). La relación de conversión dada por (9) se aplicó al resultado calculado en el presente estudio $\left(\mathrm{m}_{\mathrm{b}}=5,60\right)$ para el terremoto del 2008 en Quetame, y se obtuvo 6,0 Mw.

Recientemente, se publicó el catálogo global instrumental homogéneo en Mw (Storchak, et al., 2013), y en ese ámbito de investigación se calibraron las relaciones de conversión de magnitudes de tipo exponencial (11) o lineal-ortogonal (GOR, 12) para $\mathrm{m}_{\mathrm{b}}$ - Mw, y de tipo GOR (13) para Ms-Mw (Storchak, et al., 2012):

$$
\begin{gathered}
\mathrm{Mw}=\exp _{\mathrm{b}}^{(-4,66+0,86 \mathrm{~m})}+4,56 \quad 4,5 \leq \mathrm{m}_{\mathrm{b}} \leq 6,0 \\
\mathrm{Mw}=1,38 \mathrm{~m}_{\mathrm{b}}-1,79
\end{gathered}
$$



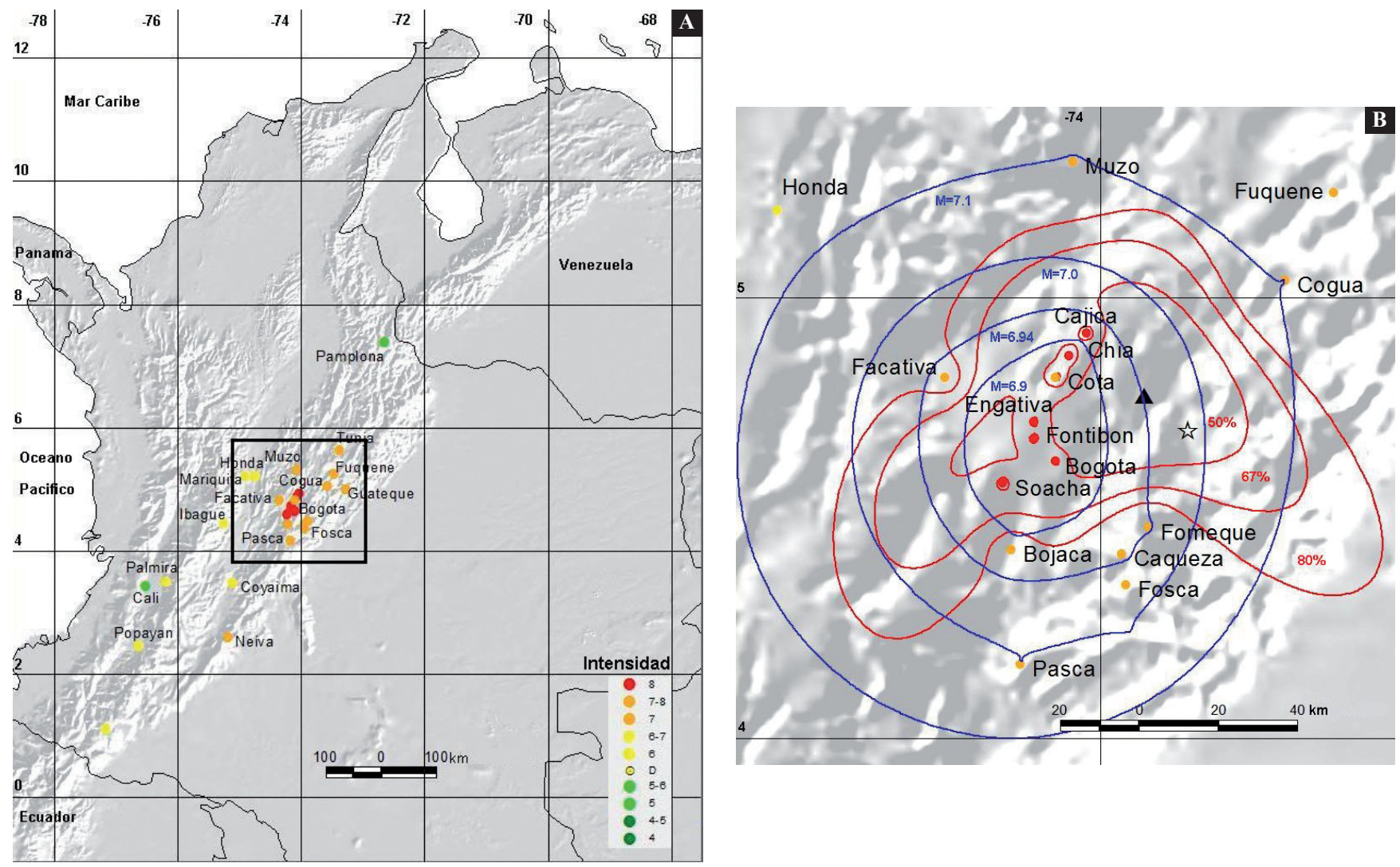

Figura 5. Terremoto del 12 de julio de 1785 en el centro de Colombia. Puntos de intensidad macrosísmica tomados de Salcedo Hurtado y Castaño Castaño (2011). Epicentro según el Ceresis (1985) (estrella vacía, 4,78 N y 73,90 O). Epicentro calculado en el presente trabajo (triangulo, 4,66 $\mathrm{N}$ y 73,90 O). Las isolíneas rojas corresponden a contornos de confianza de $\mathrm{rms}\left[\mathrm{M}_{\mathrm{I}}\right]$.

$$
\mathrm{Mw}=1,10 \mathrm{Ms}-0,67 \quad \mathrm{Ms} \geq 6,47
$$

Las relaciones (11) y (12) podrían usarse con la debida precaución cuando $\mathrm{m}_{\mathrm{b}}>6,8$ (Storchak, et al., 2012). Para el terremoto de Quetame del 2008, el valor calculado en el presente trabajo $\left(5,6 \mathrm{~m}_{\mathrm{b}}\right)$, convertido en magnitud momento usando las relaciones (11) y (12), fue de 5,7 Mw y 5,9 Mw, respectivamente. La conversión mediante el modelo GOR dado por (12) concordó con el valor de Mw dado por Global CMT.

Para el caso del terremoto histórico del 12 de julio de 1785 , la magnitud 7,0 Ms propuesta por Espinosa Baquero (2003) y la magnitud $6,9 \mathrm{~m}_{\mathrm{b}}$ propuesta en el presente estudio, se transformaron usando las relaciones (9), (10) y (13) y, como se observa, conservaron sus respectivos valores. La tabla 5 resume el ejercicio de transformación de magnitudes de origen macrosísmico $\left(\mathrm{m}_{\mathrm{b}} \mathrm{y} \mathrm{Ms}\right)$ a magnitud $\mathrm{Mw}$ usando las relaciones empíricas citadas.

El resultado obtenido en la modelación de la magnitud del sismo de Quetame a partir de datos de intensidad y usando la relación (8) con el método BW97 estuvo dentro del rango de incertidumbres con respecto al valor dado por Global CMT.
Tabla 5. Conversión de magnitudes $m_{b}$ y Ms de origen macrosísmico a magnitud $\mathrm{Mw}$

\begin{tabular}{lcc}
\hline Mw a partir de relación de conversión & $\begin{array}{c}\mathbf{2 0 0 8 . 0 5 . 2 4} \\
\mathbf{m}_{\mathbf{b}}=\mathbf{5 , 6} \\
\mathbf{p r e s e n t e} \\
\text { estudio }\end{array}$ & $\begin{array}{c}\mathbf{1 7 8 5 . 0 7 . 1 2} \\
\mathbf{m}_{\mathbf{b}}=\mathbf{6 , 9} \\
\text { presente } \\
\text { estudio }\end{array}$ \\
\hline $\mathrm{m}_{\mathrm{b}}-\mathrm{Mw}$, relación 9 (Scordilis, 2006) & 6,0 & 6,9 \\
\hline Ms - Mw, relación 10 (Scordilis, 2006) & - & - \\
$\mathrm{m}_{\mathrm{b}}-$ Mw relación 11 (Storchak, et al., 2012) & 5,7 & satura \\
\hline $\mathrm{m}_{\mathrm{b}}-$ Mw relación 12 (Storchak, et al., 2012) & 5,9 & satura \\
\hline Ms - Mw, relación 13 (Storchak, et al., 2012) & - & - \\
\hline
\end{tabular}

En los casos de sismos históricos fuertes, esta modelación podría tener inconvenientes por efecto de la saturación de la magnitud $m_{b}$ para valores altos $\left(m_{b}>6,0\right)$.

\section{Análisis DOFA y conclusiones}

Para finalizar el presente estudio, se propone un análisis de tipo DOFA (Jackson, et al., 2003) para corregir en futuros estudios las debilidades y amenazas de origen interno y 


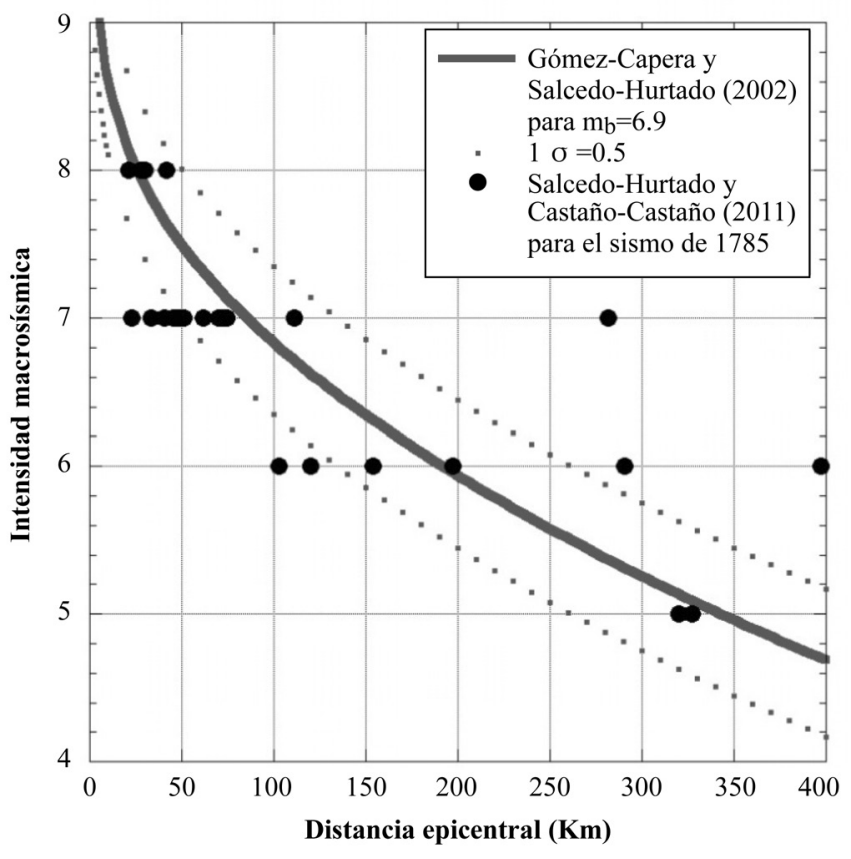

Figura 6. Intensidades macrosísmicas observadas en el terremoto del 12 de julio de 1785 comparado con la curva de atenuación de Gómez Capera y Salcedo Hurtado (2002) para una 6,9 m aprovechar las fortalezas y oportunidades de origen externo encontradas en el presente estudio, tal como se muestra en la Figura 7.

Los parámetros sismológicos (epicentro y magnitud con incertidumbre) del terremoto histórico del 12 de julio de 1785 en Colombia, se calcularon a partir de los datos de intensidad macrosísmica propuestos por Salcedo Hurtado y Castaño Castaño (2011), aplicando el algoritmo de Bakun y Wentworth (1997) y la ecuación de atenuación de la intensidad macrosísmica propuesta por Gómez Capera y Salcedo Hurtado (2002).

La modelación se validó reproduciendo los parámetros del terremoto de manera comparable con aquellos de origen instrumental. Estos resultados se consideran bastante satisfactorios a pesar de que la ecuación de atenuación utilizada fue "calibrada" en cuanto a su tiempo con datos que actualmente no se consideran modernos y son muy subjetivos, como las isosistas, y, simultáneamente, con datos instrumentales disponibles, como la magnitud de ondas internas $\mathrm{m}_{\mathrm{b}}$. Desde este punto de vista, se considera que el método usado en el presente estudio puede aplicarse sistemáticamente a otros eventos históricos registrados en los diferentes catálogos publicados, contribuyendo

\begin{tabular}{|c|c|c|}
\hline DOFA & Eje Positivo & Eje Negativo \\
\hline $\begin{array}{l}\text { Origen } \\
\text { interno }\end{array}$ & $\begin{array}{l}\text { Fortalezas } \\
\text { El método usa todos los puntos de intensidad macrosísmica } \\
\text { para el cálculo de parámetros de terremotos. } \\
\text { La localización del centro de intensidades según el método } \\
\text { de BW97 es independiente del tipo de magnitud usada en } \\
\text { la calibración de la curva de atenuación de la intensidad } \\
\text { macrosísmica. } \\
\text { El método permite obtener valores para intervalos } \\
\text { de confianza del centro de intensidades y valores de } \\
\text { incertidumbre para la magnitud calculada. }\end{array}$ & $\begin{array}{l}\text { Debilidades } \\
\text { La curva de atenuación usada no está calibrada en Mw. } \\
\text { La curva está en función de la distancia epicentral. } \\
\text { En estudios futuros se recomienda calibrar una relación } \\
\text { de atenuación en función de Mw y de la distancia } \\
\text { hipocentral para tener información explícita de la } \\
\text { profundidad de la fuente del terremoto. } \\
\text { Pueden usarse con cautela relaciones de transformación } \\
\mathrm{m}_{\mathrm{b}}-\mathrm{Mw} \text {. }\end{array}$ \\
\hline $\begin{array}{l}\text { Origen } \\
\text { externo }\end{array}$ & $\begin{array}{l}\text { Oportunidades } \\
\text { Para el terremoto de } 1785 \text {, la literatura ofrece diferentes } \\
\text { estudios macrosísmicos de diferentes autores y con } \\
\text { diferentes interpretaciones que en el futuro podrían usarse } \\
\text { para capturar la incertidumbre de origen epistémico en la } \\
\text { asignación de parámetros de terremotos históricos. } \\
\text { En la literatura se pueden encontrar simultáneamente datos } \\
\text { macrosísmicos y parámetros instrumentales de terremotos, } \\
\text { con los cuales podrían calibrarse curvas de atenuación de la } \\
\text { intensidad macrosísmica para la región andina (por ejemplo, } \\
\text { el terremoto de Quetame del 2008). }\end{array}$ & $\begin{array}{l}\text { Amenazas } \\
\text { La asignación de intensidades para terremotos } \\
\text { históricos tiene sus dificultades. Pueden encontrarse } \\
\text { discrepancias entre varios autores en cuanto a } \\
\text { los valores obtenidos en determinados sitios, lo } \\
\text { que afectaría la modelación de los parámetros del } \\
\text { terremoto. } \\
\text { La aplicación de un modelo de atenuación de } \\
\text { intensidad en función de } m_{b} \text { podría valorar } \\
\text { exageradamente la modelación de las magnitudes por } \\
\text { efecto de su saturación. }\end{array}$ \\
\hline
\end{tabular}

Figura 7. Análisis DOFA del presente estudio 
así a calcular de forma más objetiva los parámetros. Considerando que hoy se dispone de nuevos estudios macrosísmicos en el país, en el futuro se podrán proponer modelos de atenuación de la intensidad macrosísmica para Colombia en función de la magnitud Mw, así como distancias en diferentes métricas, calibradas con los datos de terremotos recientes.

\section{Conflicto de interés}

Los autores declaran que no tienen conflicto de interés.

\section{Bibliografía}

Álvarez A. (1987). Contribución al conocimiento de la sismicidad histórica en Colombia. Tesis, Universidad de los Andes, 298 p.

Álvarez-Rubio S., Kästli P., Fäh D., Sellami S. \& Giardini D. (2011). Parametrization of historical earthquakes in Switzerland, J. Seismol. 16(1): 1-24, http://dx.doi.org/10. 1007/s10950-011-9245-8

Ambraseys N.N. (1971). Value of historical records of earthquakes. Nature, 32: 375-379.

Bakun, W. H. \& C. M. Wentworth (1997). Estimating earthquake location and magnitude from seismic intensity data, Bull. Seism. Soc. Am. 87: 1502-1521.

Bakun W. H. \& Wentworth C.M. (1999). Erratum to Estimating earthquake location and magnitude from seismic intensity data, Bull. Seism. Soc. Am. 89: 557.

Bakun W.H. (2006). Estimating locations and magnitudes of earthquakes in Southern California from modified Mercalli intensities, Bull. Seism. Soc. Am. 96: 1278-1295.

Bakun W.H., Gómez Capera A.A., Stucchi M. (2011). Epistemic uncertainty in the location and magnitude of earthquakes in Italy from macroseismic data, Bull. Seismol. Soc. Am. 100: $2712-2725$.

Bakun, W. H., Flores, C. H., Ten Brink, U. S. (2012). Significant Earthquakes on the Enriquillo Fault System, Hispaniola, 1500-2010: Implications for Seismic Hazard, Bull. Seismol. Soc. Am. 102: 18-30.

Beauval C., Yepes H., Bakun W.H., Egred J., Alvarado A. \& Singaucho J-C. (2010). Locations and magnitudes of historical earthquakes in the Sierra of Ecuador (1587-1996), Geophys. J. Int. 181: 1613-1633.

Bindi D., Parolai S., Gómez-Capera A.A., Locati M., Kalmetyeva Z., Mikhailova N. (2013a). Locations and magnitudes of earthquakes in Central Asia from seismic intensity data, Journal Seismology. http://dx.doi.org/10.1007/s10950-0139392-1

Bindi D., Gómez Capera A.A., Parolai S., Abdrakhmatov K., Stucchi M \& Zschau J. (2013b). Location and magnitudes of earthquakes in Central Asia from seismic intensity data: model calibration and validation Geophys. J. Int., 192:710724; http://dx.doi.org 10.1093/gji/ggs039
Centro Regional de Sismología para América del Sur - Ceresis (1985). Catálogo de terremotos para América del Sur. Programa para la mitigación de los efectos de los terremotos en la región andina (Proyecto SISRA). Earthquake Mitigation Program, 14Vol.

Cifuentes H., Sarabia A. (2011). Sismicidad histórica en Colombia. Estudios macrosísmicos (1644-2008). Servicio Geológico Colombiano. Bogotá. Fecha de consulta: Febrero 15 de 2014. Disponible en: http://aplicaciones1. ingeominas.gov.co/sicat/html/SubProductos.aspx?Identificad or $=210012105000024413000000000$.

Choy J.E., Palme C., Guada C., Morandi M. \& Klarica S. (2010). Macroseismic Interpretation of the 1812 Earthquake in Venezuela Using Intensity Uncertainties and A Priori Fault-Strike Information. Bull. Seismol. Soc. Am., 100: 241-255.

Dimaté C., Drake L., Yepes H., Ocola L., Rendón H., Grunthal G. and Giardini D. (1999). Seismic hazard assessment in the Northern Andes (Piloto Project), Annali di Geofisica 42(6): 1039-1055.

Dorbath L., Cisternas A., Dorbath C. (1990). Assessment of the size of large and great historical earthquakes in Perú. Bull. Seism. Soc. Am. 80(3): 551-576.

Espinosa Baquero A. (2003). Historia Sísmica de Colombia (1550 -1830). Academia Colombiana de Ciencias Exactas, Físicas y Naturales - Universidad del Quindío, CD rom. Bogotá.

Gasperini P., Bernardini F., Valensise G. \& Boschi E. (1999). Defining seismogenic sources from historical felt reports. Bull. Seismol. Soc. Am. 89: 94-110.

GEM Global Large Historical Earthquake Catalogue (10001903) - GEH (2013). En Ekström, G., M. Nettles, and A. M. Dziewonski, 2004-2010: Centroid-moment tensors for 13, 017 earthquakes, Phys. EarthPlanet. Inter., 200-201, 1-9, 2012. doi:10.1016/j.pepi.2012.04.002. Fecha de consulta: Febrero 15 de 2014. Disponible en: http://www.globalquakemodel. org/what/global-projects/historical-catalogue/.

Giesecke A., Gómez Capera A.A., Leschiutta I., Migliorini E. (2004). The CERESIS earthquake catalogue and database of the Andean Region: Background, characteristics and examples of use. Annals of Geophysics. 47(2/3): 421-435.

Global Centroid Moment Tensor Project - HRVD. En Albini P., Musson R.M.W., Rovida A., Locati M., Gómez Capera A.A. and Viganò D. (2014). The Global Earthquake History. Eartquake Espectra, 30:2:607-624, Earthquake Engineering Research Institute Fecha de consulta: Febrero 15 de 2014 Disponible en: http://www.globalcmt.org/CMTsearch.html

Gómez Capera A.A. (1998). Atenuación de la intensidad sísmica para el territorio colombiano. Tesis de grado en Física, Facultad de Ciencias, Departamento de Fisica, Universidad Nacional de Colombia, Bogotá, 120p.

Gómez Capera A.A. \& Salcedo Hurtado E. de J. (2002). Leyes de atenuación de la intensidad macrosísmica en Colombia. Earth Sciences Research Journal. 6: 53-61. 
Gómez Capera A.A. \& Leschiutta I. (2004). Determining the parameters of earthquake sources in South America from macroseismic intensity data (CERESIS database). Earth Sciences Research Journal 8(1): 10-18. ISSN: 1794-6190.

Gómez-Capera A.A., García Peláez J., Chuy Rodríguez T.J., Salcedo-Hurtado E. de J., Stucchi M. (2013). Calibración y validación del algoritmo de Bakun and Wentworth para calcular localización y magnitud de terremotos históricos a partir de datos macrosísmicos en Cuba Oriental. Boletín de Geología, Universidad Industrial de Santander, Bucaramanga. 35(2): 113-127.

Grünthal G.(Ed.) (1998). European Macroseismic Scale 1998. Conseil de l'Europe, Cahiers du Centre Européen de Géodynamique et de Séismologie. Luxembourg, 100p.

Gutenberg B. \& Richter C.F. (1956). Earthquake magnitude, intensity, energy and acceleration (Second Paper). Bull. Seismol. Soc. Am. 46: 105-145.

Howell B. F. Jr. \& Schultz T.R. (1975). Attenuation of modified Mercalli intensity with distance from epicenter. Bull. Seism. Soc. Am. 63(3): 651-665.

Ingeominas (1999a). Mapa de Grandes Sismos en Colombia, 15661999, Publicación especial (poster), Bogotá.

Ingeominas (1999b). Actualización Catálogo de Sismos de Colombia para Estudios de Amenaza Sísmica, 1566 - 1998, Informe interno, Bogotá, 17pp.

Jackson S.E., Joshi A. \& Erhardt N. L. (2003). Recent Research on Team and Organizational Diversity: SWOT Analysis and Implication, Journal of Management. 29: 801-830.

Ramírez J. E., S.J. (1975). Historia de los terremotos en Colombia. Segunda edición. Instituto Geográfico Agustín Codazzi, Bogotá, 250 pp.

Salcedo Hurtado E. de J. \& Castaño Castaño A. N. (2011). Reevaluación macrosísmica del terremoto del 12 de julio de 1785 en Colombia. Boletín de Geología, Universidad Industrial de Santander, Bucaramanga. 33(2): 15-32.

Salcedo-Hurtado E. de J. \& Gómez-Capera A.A. (2013). Estudio macrosísmico del terremoto del 18 octubre del 1743 en la región central de Colombia. Boletín de Geología, Universidad Industrial de Santander, Bucaramanga. 35(1): 109-128.

Sarabia A., Cifuentes H., Dimate M. (2006). Estudio macrosísmico del sismo ocurrido el 12 de julio de 1785. Ingeominas. Bogotá. Fecha de consulta: Febrero 15 de 2014. Disponible en: http://aplicaciones1.ingeominas.gov.co/sicat/ html/Metadato.aspx?CID $=239714$

Sarabia A.M. \& Cifuentes H. G. (2009). Análisis macrosísmico del sismo del 24 de mayo de 2008 en Quetame. Ingeominas, Bogotá, 44p. Fecha de consulta: Febrero 15 de 2014. Disponible en: http://aplicaciones1.ingeominas.gov.co/sicat/ html/Metadato.aspx?CID $=239800$

Sarabia Gómez A.M., Cifuentes Avendaño H. G., Robertson K. (2010). Análisis histórico de los sismos ocurridos en 1785 y en 1971 en el centro de Colombia, Revista Colombiana de Geografia. 19:153-162.ISSN:0121-215X.

Scordilis E.M. (2006). Empirical global relations converting Ms and $\mathrm{mb}$ to moment magnitude. Journal Seismology. 10:225236. http://dx.doi.org/10.1007/s10950-006-9012-4

Servicio Geológico Colombiano - Universidad Nacional de Colombia. (SGC-UN) (2012). Sistema de información de sismicidad histórica de Colombia. Fecha de consulta: Febrero 15 de 2014. Disponible en línea: http://agata. ingeominas.gov.co:9090/SismicidadHistorica/.

Storchak D. A., Di Giacomo D., Bondár I., Harris J., Engdahl E.R., Lee W.H.K., Villaseñor A., Bormann P. \& Ferrari G. (2012). ISC-GEM Global Instrumental Earthquake Catalogue (19009-2009), Final Scientific Report, 99p. Fecha de consulta: Febrero 15 de 2014. Disponible en línea: http://www.globalquakemodel.org/what/seismic-hazard/ instrumental-catalogue/

Storchak D. A., Di Giacomo D., Bondár I., Engdahl E.R., Harris J., Lee W.H.K., Villaseñor A. \& Bormann P. (2013). Public release of the ISC-GEM Instrumental Earthquake Catalogue (1900-2009). Seismological Research Letters. 84(5):810-815, http://dx.doi.org/10.1785/0220130034 